\title{
An efficient algorithm to calculate intrinsic thermoelectric parameters based on Landauer approach
}

\author{
Abhijeet Paul · Shuaib Salamat · Changwook \\ Jeong • Gerhard Klimeck • Mark Lundstrom
}

Received: date / Accepted: date

\begin{abstract}
The Landauer approach provides a conceptually simple way to calculate the intrinsic thermoelectric (TE) parameters of materials from the ballistic to the diffusive transport regime. This method relies on the calculation of the number of propagating modes and the scattering rate for each mode. The modes are calculated from the energy dispersion $(\mathrm{E}(\mathrm{k}))$ of the materials which require heavy computation and often supply energy relation on sparse momentum $(\mathrm{k})$ grids. Here an efficient method to calculate the distribution of modes $(\mathrm{DOM})$ from a given $\mathrm{E}(\mathrm{k})$ relationship is presented. The main features of this algorithm are, (i) its ability to work on sparse dispersion data, and (ii) creation of an energy grid for the DOM that is almost independent of the dispersion data therefore allowing for efficient and fast calculation of TE parameters. The effect of k-grid sparsity on the compute time for DOM and on the sensitivity of the calculated TE results are provided. The algorithm calculates the TE parameters within $5 \%$ accuracy when the K-grid sparsity is increased up to $60 \%$ for all the dimensions (3D, 2D and $1 \mathrm{D})$. The time taken for the DOM calculation is strongly influenced by the transverse $\mathrm{K}$ density ( $\mathrm{K}$ perpendicular to transport direction) but is almost independent of the transport $\mathrm{K}$ density (along the transport direction). The DOM and TE results from the algorithm are bench-marked with, (i) analytical calculations for parabolic bands, and (ii) realistic electronic and phonon results for $B i_{2} T e_{3}$.
\end{abstract}

Keywords Landauers method - Thermoelectricity · Electronic structure · Phonons · Density of Modes

\section{Introduction}

Thermoelectricity provides an attractive and a clean way of converting waste heat into electricity. There have been a lot of efforts to improve the efficiency of thermoelectric (TE) devices. Solid-state TE devices are aggressively pursued both in the industry

Abhijeet Paul, Shuaib Salamat, Changwook Jeong, Gerhard Klimeck and Mark Lundstrom School of Electrical and Computer Engineering and

Network for Computational Nanotechnology

Purdue University, West Lafayette, USA 47907

Tel.: 1-765-404-3589 E-mail: abhijeet.rama@gmail.com 
and research due to their advantages such as, (i) compactness, (ii) resistance to wear and tear, and (iii) portability. Thermoelectric efficiency $(Z T)$ improvements need very careful engineering designs and optimization in terms of, (i) materials [1,2,3,4, (ii) structures like superlattices, nanocomposities, etc. [5, 6, 7, 8, and (iii) devices [9, 10, 11, 12,13. With so many design parameters it is extremely difficult to experimentally test every possible combination. At this point computer modeling plays a very significant role in designing and optimizing TE devices from material to the system level [14,15, 16. The present work focuses on the calculation of the TE transport parameters using the material energy dispersion as shown in Fig. 1

In the present work we focus on the calculation of the material properties involved in the calculation of $Z T$. The value of $Z T$ and the thermoelectric power-factor $(P F)$ for a material are given by $17,18,19$,

$$
\begin{aligned}
& Z T=\frac{G \cdot S^{2}}{\kappa_{e}+\kappa_{l}} \cdot T \quad[\text { unitless }] \\
& P F=G \cdot S^{2} \quad\left[W / K^{2} m^{d-1}\right]
\end{aligned}
$$

where $G, S, \kappa_{e}$ and $\kappa_{l}$ are the electronic conductivity, electronic Seebeck coefficient, electronic thermal conductivity and lattice thermal conductivity, respectively. The term ' $d$ ' is the dimensionality of the conductor. All the TE parameters depend on the electronic and lattice properties of the material.These material properties are strongly coupled and an improvement in one of the coefficients may degrade the other [19.

The Boltzmann transport equation (BTE) [19,20, has been the most commonly used method to calculate the TE material parameters. However, with reduced dimensionality of the TE materials (like nanodots, nanowires, etc) the application of the Landauer approach 21,22] for calculating the TE parameters has gained a lot of attention [17, 18, 23,24,25,26,27 due to the simplicity of the approach. The Landauer approach is applicable from the ballistic to the diffusive regime of transport for nanostructures. This model is insightful for understanding the impact of dimensionality on TE parameters too [18.

At the core of the Landauer approach is the calculation of distribution of modes (DOM) 17, 23, 18, which is similar to the transport distribution function (TDF) used in the BTE model 28 as shown in Refs. 17, 23 for both electrons and phonons. The DOM represents the number of conducting channels available for the carriers, like electrons or phonons, at a given energy. From computational aspect, most of the previous work using Landauer's approach relied heavily on very fine $\mathrm{E}(\mathrm{k})$ calculations and then calculating the DOM by band-counting method 17 as shown in Fig. 2. The BTE methods use the reduced Brillouin Zone (BZ) integration schemes 29,30] to calculate the TE parameters. However, these approaches too depend on a fine momentum mesh for numerical integrations. The computation of the dispersion relations in novel materials require significant computational resources and in general delivers results on momentum meshes that are not dense enough to derive a complete DOM or TDF.

To overcome the above mentioned computational challenges an efficient algorithm to calculate the DOM (used in the Landauer model) from a given $\mathrm{E}(\mathrm{k})$ is outlined in this work. The present method has two advantages over the previous band-counting methods, which are, (i) the energy dispersion $(\mathrm{E}(\mathrm{k})$ ) can be relatively sparse, and (ii) the energy grid for the DOM and the $\mathrm{E}(\mathrm{k})$ does not have to be identical. Overall compute time for the calculation of TE parameters is reduced in two steps, (i) relatively 
little compute time is needed to calculate the DOM from the sparse energy dispersion, and (ii) the sparse DOM energy grid further reduces calculation time of the TE parameters $\left(G, S, \kappa_{e}\right.$, and $\left.\kappa_{l}\right)$.

The present work is divided in the following sections. The basic TE theory in the linear transport regime is outlined in Sec. 2.1 The generic algorithm for DOM calculation is presented in Sec. 2.2 with specific changes required for electrons in Sec. 2.3 and for phonons in Sec. 2.4 Discussion on the transmission calculation is provided in Sec. 2.5 The results section provides the k dependent sensitivity analysis in Sec. 3.1 and the timing analysis of the algorithm in Sec. 3.2 Comparison and verification of the TE parameters, calculated using the algorithm, with published results are provided in Sec. 3.4 The summary of the work is outlined in Sec. 4

\section{Theory and methodology}

In this section the calculation of the TE parameters and the details of the algorithms are outlined.

2.1 Thermoelectric parameters in the linear transport regime

The $Z T$ of a material at a temperature, $T$, is based on the calculation of the intrinsic material properties which include both the electronic and the lattice properties (see Eq. (1)). The electronic transport parameters are obtained using the Landauer approach in the zero current limit [17,23] as,

$$
\begin{aligned}
G & =\frac{2 q^{2}}{h} \cdot I_{0} \quad\left[\Omega^{-1} m^{d-1}\right] \\
S & =-\left[k_{B} / q\right] \cdot\left[I_{1} / I_{0}\right] \quad[V / K] \\
\kappa_{e} & =\left[\frac{\left(2 T k_{B}^{2}\right)}{h}\right] \cdot\left[I_{2}-\left(I_{1}^{2} / I_{0}\right)\right] \quad\left[W / m^{d-1} K\right] \\
I_{j} & =\int_{E_{\min }}^{E_{\max }}\left[\frac{\left(E-E_{F}\right)}{k_{B} T}\right]^{j} \cdot \mathcal{T}(E) \cdot \mathcal{M}(E) \cdot \frac{-\partial \mathcal{F}_{F D}}{\partial E} \cdot d E,
\end{aligned}
$$

where $I_{j}$ is the jth order energy moment integration around the Fermi Level $\left(E_{F}\right)$. The terms $q, k_{B}$ and $h$ are the electronic charge, Boltzmann constant, and Planck's constant, respectively. In the quantity $I_{j}$ the terms $\mathcal{M}(\mathrm{E}), \mathcal{T}(\mathrm{E})$, and $\mathcal{F}_{F D}$ are the distribution of modes (DOM) at energy $E$, transmission at energy $E$ and the FermiDirac distribution function, respectively.

The lattice thermal conductivity $\left(\kappa_{l}\right)$ can be calculated using the Landauer's model as 23, 26, 27,

$$
\begin{aligned}
\kappa_{l}(T)= & \hbar P_{1} \quad\left[W / m^{d-1} K\right] \\
P_{j}= & \int_{\omega_{\min }}^{\omega_{\max }} \mathcal{T}(\omega) \cdot \mathcal{M}(\omega) \cdot \omega^{j} . \\
& \frac{\partial}{\partial T}\left[\left(\exp \left(\frac{\hbar \omega}{k_{B} T}\right)-1\right)^{-1}\right] \cdot d \omega
\end{aligned}
$$


Table 1 Dimensionality of structure and dependence on ' $K$ ' vectors

\begin{tabular}{|l|c|c|c|c|l|}
\hline $\begin{array}{l}\text { Structure } \\
\text { (dimension) }\end{array}$ & $\begin{array}{c}\text { Periodic } \\
\text { dim. (P) }\end{array}$ & $\begin{array}{c}\text { Confined } \\
\text { dim. (C) }\end{array}$ & $K_{\perp}$ & $K_{\|}$ & $\mathrm{K}$ \\
\hline Wires (1D) & 1 & 2 & 0 & 1 & {$\left[K_{\|}\right]$} \\
Films (2D) & 2 & 1 & 1 & 1 & {$\left[K_{\perp}, K_{\|}\right]$} \\
Bulk (3D) & 3 & 0 & 2 & 1 & {$\left[K_{\perp}^{1}, K_{\perp}^{2}, K_{\|}\right]$} \\
\hline
\end{tabular}

where $P_{j}$ is the jth order phonon energy integration. The terms $\mathcal{M}(\omega)$ and $\mathcal{T}(\omega)$ are the distribution of modes, and the transmission of the modes at a phonon frequency $\omega$, respectively.

Equations (6) and (7) show that the calculation of any transport parameter within the Landauer model depend on two quantities, (i) the distribution of modes $(\mathcal{M})$ and, (ii) the transmission of the modes $(\mathcal{T})$. The DOM depends only on the energy dispersion of the carriers in the material whereas the transmission is controlled by the dispersion and the scattering mechanisms of the carrier. The advantage of the Landauer model lies in the separation of the transport kernel into two parts that can be solved using parallel computer programming leading to a faster and efficient calculation of the transport parameters. In the next part the details of the algorithm to efficiently calculate the DOM from a given energy dispersion is outlined.

\subsection{Distribution of Modes calculation}

The step by step procedure for the calculation of DOM (applicable to both electrons and phonons) is given below,

1. Obtain the energy dispersion of an $d$ dimensional, where $d=1,2$ or 3 , periodic material. The momentum vector ' $K$ ' can be decomposed into two components, (i) along the transport direction denoted by $K_{\|}$, and (ii) in the direction perpendicular to the transport direction denoted by $K_{\perp}$ depending on the dimensionality of the conductor as shown in Table 1 .

2. For each combination of $K_{\perp}$, a $1 \mathrm{D} E-K_{\|}$is obtained which is used for mode counting. The energy grid for the DOM (EGD) is created based on the 1D $E-K_{\|}$ for all the $K_{\perp}$. This energy grid does not have to be identical to the energy values from the $E(k)$ data. The details of choosing the energy limits for the electrons and phonons are outlined in Sec 2.3 and Sec. 2.4 respectively. The energy grid is chosen so as to provide a reasonable compromise between the computation time and the accuracy of the results.

3. For a $1 \mathrm{D} E-K_{\|}$, the group velocity $\left(v_{\text {grp }}\right)$ is calculated to find out the regions of monotonic variation in the energy with $K_{\|}$. Only positive $K_{\|}$are considered since the $E-K_{\|}$relations are symmetric. The +ve half group velocity is calculated as,

$$
v_{g r p}=\frac{1}{\hbar} \frac{\partial E(K)}{\partial K_{\|}}
$$

The monotonic velocity regions, $\mathrm{R} 1$ and $\mathrm{R} 2$, for an example $E(k)$ are shown in Fig. 3.

4. These monotonic velocity regions are then used for counting modes. The EGD points are calculated by the interpolation of the $E-K_{\|}$data points using the $V_{\text {grp }}$. The details for calculating the energy nodes on the EGD is shown in Fig. 4 
As a by-product of the calculation the carrier velocity is also obtained which can be used for other calculations such as the mean free path.

5. The modes from each of the 1D $E-K_{\|}$are then integrated over all the $K_{\perp}$ and divided by the unit-cell area $\left(A_{u c}\right)$ to obtain the total DOM.

The present algorithm has the advantage that the original $E-K_{\|}$can be sparse compared to the energy grid on which the DOM is calculated since the monotonic regions of $E-K_{\|}$allow to interpolate the dispersion data to be used for DOM and velocity calculations. The strength of the algorithm to obtain the DOM for different K-grid densities is shown in Fig. 5. Since the algorithm can work on sparse dispersion data, the time to obtain the total modes is also reduced.

The present algorithm assumes that all the $K$ vectors for a given energy dispersion are orthogonal. This assumption has both advantages and disadvantages. Since the $K_{\|}$ and $K_{\perp}$ are separated, this allows for parallel computation of modes for each $K_{\perp}$ set. This leads to computational speed-up. This aspect of the algorithm can be inspected as a future work. The interpolation in the $\mathrm{E}(\mathrm{k})$ is always done along $K_{\|}$but not along $K_{\perp}$. This puts a limitation on the sparsity of the $K_{\perp}$ grid. A very sparse $K_{\perp}$ grid will result in erroneous DOM calculation. The over-all E(k) should not be too sparse either such that the original features of the dispersion are lost. In that case velocity interpolation will give erroneous results. The sensitivity of the TE results on the K-grid sparsity and the compute time for DOM are discussed in Sec. 3.1 and Sec 3.2 respectively.

Apart from the general steps adopted for the calculation of the DOM for both electrons and phonons, some special care in selecting the energy ranges for both, involved in Eq. (6) and (8), must be taken.

\subsection{Energy range: electron transport}

Real materials are characterized by many different electronic bands. However, not all these bands contribute to the electron transport and an energy range around the Fermilevel $\left(E_{F}\right)$ needs to be selected carefully. To obtain an expedient but good approximate solution the energy cut-offs $\left(E_{\min }, E_{\max }\right)$ are chosen such that the integral values for the transport parameters ( Eq. (33)-(6p) do not show any variation. The bounds for the energy grid (Eq. 6) of the DOM is obtained as follows,

$$
\begin{array}{lll}
E_{\max }=E c & \text { or } & \min \left[\max \left[E\left(K_{\|}\right) \forall K_{\perp}\right]\right] \\
E_{\min }=E v & \text { or } \quad \max \left[\min \left[E\left(K_{\|}\right) \forall K_{\perp}\right]\right]
\end{array}
$$

where $\min (\max )$ represent the minimum (maximum) value in a given numerical array. The terms $E c$ and $E v$ define the conduction band minima (CBM) and the valence band maxima (VBM), respectively as shown in Fig. 6. Our calculations show that in order to obtain correct results, the $E_{F}$ value can vary between the following limit,

$$
E_{\min }+10 k_{B} T \leq E_{F} \leq E_{\max }-10 k_{B} T
$$

where $T$ is the temperature. The choice of $10 k_{B} T$ is chosen since this gives a good range where the integrals involved in the TE parameter calculations become invariant to the choice of energy grid as shown in Fig. 7 
2.4 Energy range: lattice transport

The lattice kernel calculations do not depend on any kind of Fermi-level. Unlike the electron bands, the phonon bands are always within a fixed energy range with a varying number of sub-bands for different dimensional structures 31,32. Also there is no negative phonon energy dispersion in stable semi-conductor structures [33, hence the energy grid of the DOM always contains positive values. The energy limit for the lattice kernel is chosen as follows,

$$
\begin{aligned}
\omega_{\min }=0 \quad \text { or } & \text { User defined } \\
\omega_{\max }=\Omega_{\max } & \text { or } \quad \text { User defined, }
\end{aligned}
$$

where $\Omega_{\max }$ is the maximum energy limit of the phonon dispersion.

\subsection{Transmission calculation}

For ballistic transport of electrons or phonons the transmission $(\mathcal{T}(\mathrm{E}))$ of all the modes is 1 . However, in reality carriers undergo a lot of scattering which depends on the dimensionality of the system, doping, temperature, etc. This reduces the transmission of the modes below 1 . For a conductor of length $L, \mathcal{T}(E)$ is given by 17,23 ,

$$
\mathcal{T}(E)=\frac{<\lambda(E)>}{L+<\lambda(E)>}
$$

where $\langle\lambda(E)\rangle$ is the carrier mean free path (MFP) obtained by the summing over all allowed $k$ points at energy $E$. When $L>>$ MFP (diffusive limit) then Eq. 15. can be approximated as,

$$
\mathcal{T}(E) \approx \frac{<\lambda(E)>}{L}
$$

All the scattering mechanisms present in a system are lumped in the 'mean free path'. The energy dependence of the MFP can be broadly classified into two categories, (i) constant MFP (no energy dependence), and (ii) energy dependent MFP. For some common scattering mechanisms like ionized impurity, acoustic phonon, etc, $\langle\lambda(E)\rangle$ can be expressed in a power law form as $\langle\lambda(E)\rangle=\lambda_{0}\left[E /\left(k_{B} T\right)\right]^{r}$, where $E$ is the kinetic energy of the carrier, $\lambda_{0}$ is a constant and ' $r$ ' is a characteristic exponent describing a specific scattering process [17.

In most of the BTE calculations the scattering time $\left(\tau_{\text {scat }}\right)$ is used instead of the MFP. Again for $\tau_{\text {scat }}$ the energy dependence are of two types, (i) energy independent (constant $\tau_{\text {scat }}$ ), and (ii) energy dependent. The constant $\tau_{\text {scat }}$ case is physically hard to justify since it is well known that particles scatter to/from different energy states at a different rate [17]. The connection of the scattering time to the MFP is given as [17,

$$
<\lambda(E)>=2 \cdot \frac{\sum_{K} v_{\|}^{2}(K, E) \cdot \tau_{s c a t}(K, E)}{\sum_{K}\left|v_{\|}(K, E)\right|}
$$

Here the summation is over all the $K$ states at a given energy $E$. If the scattering time is assumed isotropic in $K$ then the MFP is given as, 


$$
<\lambda(E)>=2 \cdot\left[\frac{\sum_{K} v_{\|}^{2}(K, E)}{\sum_{K}\left|v_{\|}(K, E)\right|}\right] \cdot \tau_{\text {scat }}(E)
$$

In the present algorithm, the MFP can be calculated efficiently since the velocities are already obtained as a by-product during the DOM calculation. In the case of a constant scattering time, the energy dependence of the MFP is derived purely from the electronic or phonon energy dispersion.

\section{Results and Discussion}

In this section we provide the results on the dependence of calculated TE parameters on the sparsity of the K-grid using the algorithm. The timing analysis is provided to give an idea about the total speed up with K-grid reduction and which part of the calculation consumes the maximum compute time. Also the comparison of the DOM and TE parameters, calculated from the algorithm, with analytical expressions and realistic material dispersions are provided.

\subsection{Sensitivity analysis: How robust is the algorithm?}

To understand the strengths and limitations of the algorithm, K-grid sensitivity tests are performed using parabolic $\mathrm{E}(\mathrm{k})$ dispersions for $3 \mathrm{D}, 2 \mathrm{D}$ and $1 \mathrm{D}$ cases. The parameters used for the generation of the parabolic bands are shown in Table 2 .

The TE parameters like $\mathrm{G}$ and $\mathrm{S}$ are calculated using the parabolic $\mathrm{E}(\mathrm{k})$. The k-grid density variation introduces numerical error in the $\mathrm{S}$ and $\mathrm{G}$ calculation. The percentage error in the maximum power factor $\left(P F_{\max }\right)$ is related to the error in $S$ and $G$ by the following relation,

$$
\frac{\Delta P F_{\max }}{P F_{\max }}=2 \cdot \frac{\Delta S}{S}+\frac{\Delta G}{G},
$$

where $\Delta \mathrm{S} / \mathrm{G}$ are the variations in Seebeck coefficient and electronic conductivity, respectively. The final fluctuation in the $P F_{\max }$ depends on the sign of $\Delta \mathrm{S} / \mathrm{G}$. However, all the fluctuation plots are shown for the absolute value of the errors.

To start the sensitivity analysis, first a base K-grid is chosen. A K-grid with 100 points in each direction $\left(-\pi / a_{0}\right.$ to $\left.\pi / a_{0}\right)$ is found to be sufficient to provide stable results. A finer K-grid does not change the final results by more than $0.5 \%$ for any of the calculated TE values. Three different types of studies were performed to determine the sensitivity of the algorithm to reduction in $K_{\|}$and $K_{\perp}$,

- Case A: Keep $K_{\perp}$ fixed at 100 grid points and reduce $K_{\|}$down to $\sim 60 \%-80 \%$.

- Case B: Keep $K_{\|}$fixed at 100 grid points and reduce $K_{\perp}$ down to $\sim 60 \%-80 \%$.

- Case C: Reduce both $K_{\|}$and $K_{\perp}$ down to $\sim 60 \%-80 \%$.

For the sake of brevity only the 2D TE error analysis results are shown. Other dimensions (3D and $1 \mathrm{D})$ show similar results and the outcomes are similar. For the 1D system only case $\mathrm{C}$ is applicable since in these systems only $K_{\|}$is the free momentum direction. The other two directions are geometrically confined as shown in Table 1 . 
Table 2 Parameters used for the generation of parabolic bands

\begin{tabular}{|l|c|c|c|c|c|c|}
\hline $\begin{array}{l}\text { Structure } \\
\text { (Dim) }\end{array}$ & $\begin{array}{c}m_{\|}^{*} \\
\times m_{0}\end{array}$ & $\begin{array}{c}m_{\perp 1}^{*} \\
\times m_{0}\end{array}$ & $\begin{array}{c}m_{\perp 2}^{*} \\
\times m_{0}\end{array}$ & $\begin{array}{c}E_{c} \\
\mathrm{eV}\end{array}$ & $\begin{array}{c}E_{v} \\
\mathrm{eV}\end{array}$ & $\begin{array}{c}a_{0} \\
\mathrm{~nm}\end{array}$ \\
\hline Wires (1D) & 1 & - & - & 0.2 & -0.2 & 1 \\
Films (2D) & 1 & 1 & - & 0.2 & -0.2 & 1 \\
Bulk (3D) & 1 & 1 & 1 & 0.2 & -0.2 & 1 \\
\hline
\end{tabular}

Sensitivity Analysis: Case A

The reduction in $K_{\|}$down to $60 \%$ results in less than $1 \%$ variation in $S$ and $\sim 6 \%$ variation in $G$ as shown in Fig. 8a. The corresponding fluctuation in the $P F$ is around $5 \%$ as shown in Fig. 8p. The Fermi-level, at which the maximum PF is extracted, however remains unchanged. The fluctuation in the TE parameters arises only from the fluctuation in the DOM. Thus, the present DOM calculation method is quite robust to reductions in $K_{\|}$given the $K_{\perp}$ has good mesh density.

Sensitivity Analysis: Case B

The reduction in $K_{\perp}$ down to $60 \%$ results in less than $2 \%$ variation in $S$ and $\sim 12 \%$ variation in $G$ as shown in Fig. 9 a. The maximum fluctuation in $\mathrm{PF}$ is around $10 \%$ as shown in Fig. 9p. The Fermi-level $\left(E_{F}\right)$ at which the maximum $P F$ is extracted shows a maximum variation of $\sim 2.5 \%$. In this case, the fluctuation in the TE parameters arises from the fluctuation in, (i) the DOM, and (ii) the $E_{F}$. The present DOM algorithm is sensitive to variations in $K_{\perp}$.

Sensitivity Analysis: Case C

The reduction in all the $K$ points down to $60 \%$ results in less than $5 \%$ variation in $S$ and $\sim 13 \%$ variation in $G$ as shown in Fig. 10a. The maximum fluctuation in $P F$ is around $10 \%$ as shown in Fig. 10 . The $E_{F}$ at which the maximum $P F$ is extracted shows a maximum variation of $\sim 2.5 \%$. Thus, the fluctuation in the TE values arises from the fluctuation in, (i) the DOM, and (ii) the $E_{F}$. This case has almost similar $\mathrm{K}$-grid sensitivity as case B, again showing that the present DOM algorithm is sensitive to variations in $K_{\perp}$.

\subsection{Timing analysis}

The present algorithm can calculate the TE parameters within reasonable error limits as shown in the previous section. Another obvious question that arises is how much computational speed-up can be achieved. The time to calculate the DOM for the three cases presented in the previous section is analyzed for $3 \mathrm{D}, 2 \mathrm{D}$ and $1 \mathrm{D}$ structures.

As the $K$ density along all the directions is reduced, the speed up for each dimension is different. For the $3 \mathrm{D}$ system, the time required goes up with total number of K-points $(N K)$ with a power of $1.46\left(N K^{1.46}\right)$. For the $2 \mathrm{D}$ case the power law is $N K^{0.48}$ and for $1 \mathrm{D}$ case the time taken is almost constant (in the given $N K$ range). The algorithm takes roughly 900 seconds for 1 million K-points $(100 \times 100 \times 100)$ for $3 \mathrm{D}$ case on nanoHUB.org workspace [34. For the $2 \mathrm{D}$ case the time taken for ten thousand K-points $(100 \times 100)$ is 
Table 3 Summary of sensitivity and timing analysis

\begin{tabular}{|c||ccccccc||c|}
\hline Dimension & K-grid & \multicolumn{1}{c|}{ Max. K } & \multicolumn{3}{c|}{ Max. error (\%) } & \multicolumn{2}{c|}{ Good $E(K)$} & DOM speed up \\
& direction & reduction $(\%)$ & $\mathrm{S}$ & $\mathrm{G}$ & $\mathrm{PF}$ & $E_{F}$ & sparsity & $(60 \% \mathrm{~K}$ red.) \\
\hline 3D & $K_{\|}$ & 80 & -4.41 & 3.02 & -5.65 & 4.14 & $<70 \%$ & $1.02 \times$ \\
\hline 3D & $K_{\perp}$ & 64 & -5.3 & -4.45 & -11.49 & 1.02 & $<50 \%$ & $8.4 \times$ \\
\hline 3D & All-K & 66 & -5.3 & 15.2 & 5.9 & 5.2 & $<50 \%$ & $3.6 \times$ \\
\hline \hline 2D & $K_{\|}$ & 60 & -0.4 & 6 & 5 & 0 & $<60 \%$ & $1.1 \times$ \\
\hline 2D & $K_{\perp}$ & 60 & -0.32 & -9.30 & -9.95 & 1.18 & $<50 \%$ & $1.8 \times$ \\
\hline 2D & All-K & 58 & 4.2 & -13.3 & -4.4 & 1.18 & $<50 \%$ & $1.5 \times$ \\
\hline 1D & All-K & 80 & 4 & 13.2 & 20 & 3.8 & $<70 \%$ & $1.05 \times$ \\
\hline
\end{tabular}

nearly 2 seconds and for $1 \mathrm{D}$ case the time taken is roughly 0.1 second for $100 \mathrm{~K}$-points. All these results are shown in Fig. 11

For the cases of $2 \mathrm{D}$ and $3 \mathrm{D}$, the algorithm requires different compute times with reductions in K-points along both the transport and the transverse direction. The compute time for the DOM $\left(t_{D O M}\right)$ is roughly independent of the K-point reduction in transport direction for both the 2D and 3D cases (Fig. 12). However, for a $60 \%$ reduction in $K_{\perp}$, the $2 \mathrm{D}$ case shows a $t_{D O M}$ speed up factor of $\sim 2$ (Fig. 12 a). While for the $3 \mathrm{D}$ case, a speed up factor of 6 is observed (Fig. 12 b). A reduction in all K-points along all directions show a similar speed up (Fig. 12). Thus, the present algorithm shows a good speed up with $K_{\perp}$ point reduction.

\subsection{Discussion: Algorithm aspects}

The TE sensitivity analysis and $t_{D O M}$ speed up reveal that the algorithm to calculate the DOM is more sensitive to the $K_{\perp}$ points compared to the $K_{\|}$points. A reasonable reduction in $K_{\perp}$ must be chosen in order to optimize the compute time and to obtain reasonably stable TE parameter values. A summary of all the analysis is provided in Table 3 This table also provides the limits for reduction in $\mathrm{K}$ points in the $\mathrm{E}(\mathrm{k})$ data-set to obtain TE parameters within a $10 \%$ error margin. In most of the cases a $50 \%$ reduction in K-points is easily achievable without a big penalty on the calculated TE values. The sensitivity analysis presented here is for parabolic bands, however, the general features of the algorithm remain quite similar even for the dispersions of real materials which are more complex than parabolic bands. Similar conclusions are obtained for the phonon dispersions.

\subsection{Calculation of the TE parameters}

The final verification of the algorithm is done by calculating the TE parameters for (i) the parabolic bands in $3 \mathrm{D}, 2 \mathrm{D}$, and $1 \mathrm{D}$ cases, and (ii) bulk $B i_{2} \mathrm{Te}_{3}$.

\section{Parabolic Bands}

The parameters used for the electronic energy dispersion are shown in Table 2 For all dimensions the number of energy points in the DOM (EGD) is kept constant at 500. The analytical results for the modes and TE parameters are obtained from Ref. [17] and 18 . The number of modes for all three dimensions compare very well ( $\leq 4 \%$ error) 
with the analytical results as shown in Fig. 13 Using the modes, the TE values are calculated. Only the 2D case is shown in Fig. 14. The agreement with the analytical calculations is very good with around $1 \%$ error in the numerical values. The $3 \mathrm{D}$ and $1 \mathrm{D}$ cases also show very good agreement with the analytical calculations. Thus, the algorithm provides accurate results for the electronic TE parameters.

Realistic Bands: Bulk $\mathrm{Bi}_{2} \mathrm{Te}_{3}$

As a next step of verification, the algorithm is tested for the calculation of TE parameters for $\mathrm{Bi}_{2} \mathrm{Te}_{3}$. The same Tight-binding electronic dispersion 35] is used for the calculation of the DOM as used in Ref. 17. The agreement with the published DOM results is very good (within $1 \%$ error) as shown in Fig. 15 . Using the DOM, the $S$ and $G$ are also calculated which are used to obtain the $P F$. The calculated $P F$ again shows a very good agreement with the published theoretical result 17] as well as with the experimental data 36 as shown in Fig. 15 p. The same calculation is also performed for the lattice thermal conductivity of bulk $B i_{2} T e_{3}$. The phonon dispersion is obtained using GULP [37] as provided in Ref. [23]. The agreement of the calculated phonon modes with the published theoretical result 23 is very good (Fig. 16a). Also the lattice thermal conductivity calculated using the method provided in Ref. 23] gives a very good agreement with the experimental value [38. Thus, the present algorithm provides accurate $\mathrm{TE}$ values for real materials too.

\section{Summary and Outlook}

An efficient algorithm to calculate the electron and phonon modes in any dimension is presented. The algorithm provides an efficient implementation of a TE parameters calculation scheme based on the Landauer's approach and will be extremely useful in readily and accurately evaluating the existing as well as new thermoelectric materials. The algorithm is sensitive to the transverse $\mathrm{K}$ point density in the $\mathrm{E}(\mathrm{k})$ relation both in terms of the final TE calculations as well as the compute time. A proper optimization of the K-point reduction is provided to allow for fast and accurate TE parameter calculations. The results from the algorithm are also bench-marked with analytical and real material TE parameter values. This algorithm will be useful for developing computer programs to evaluate the TE performance of new and artificial materials in the future.

\section{Acknowledgments}

The authors would like to acknowledge the financial support from FCRP-MSD under Semiconductor Research Corporation (SRC), Nano Research Initiative (NRI) under Midwest Institute for Nanoelectronics Development (MIND) and National Science Foundation (NSF). Computational resources provided by nanoHUB.org, a portal funded by NSF under the Network for Computational Nanotechnology (NCN), is also acknowledged. 


\section{References}

1. J. P. Heremans, V. Jovovic, E. S. Toberer, A. Saramat, K. Kurosaki, A. Charoenphakdee, S. Yamanaka, and G. J. Snyder, "Enhancement of thermoelectric efficiency in PbTe by distortion of the electronic density of states," Science, vol. 321, pp. 554-557, 2008.

2. G. J. Snyder, M. Christensen, E. Nishibori, T. Caillat, and B. B. Iversen, "Disordered zinc in Zn4Sb3 with phonon-glass and electron-crystal thermoelectric properties," Nature Materials, vol. 3, pp. 458-463, 2004.

3. G. J. Snyder, J. R. Lim, C.-K. Huang, and J. P. Fleurial, "Thermoelectric microdevice fabricated by a MEMS-like electrochemical process," Nature Material, vol. 2, pp. 528-531, 2003.

4. K. Ahn, M. K. Han, J. Q. He, J. Androulakis, S. Ballikaya, C. C. Uher, V. P. Dravid, and M. G. Kanatzidis, "Exploring Resonance Levels and Nanostructuring in the PbTeCdTe System and Enhancement of the Thermoelectric Figure of Merit," Journal of the American Chemical Society, vol. 132, pp. 5227-5235, 2010.

5. A. Minnich, H. Lee, X. Wang, G. Joshi, M. Dresselhaus, Z. Ren, G. Chen, and D. Vashaee, "Modeling study of thermoelectric SiGe nanocomposites," Physical Review B, vol. 80, p. $155327,2009$.

6. M. S. Dresselhaus, G. Chen, M. Y. Tang, R. G. Yang, H. Lee, D. Z. Wang, J. P. Ren, Z. F. J. P. Fleurial, and P. Gogna, "New directions for low-dimensional thermoelectric materials," Advanced Materials, vol. 19, pp. 1043-1053, 2007.

7. M. G. Kanatzidis, "Nanostructured Thermoelectrics: The New Paradigm?" Chemistry of Materials, vol. 22, pp. 648-659, 2010.

8. Y. Lan, A. J. Minnich, G. Chen, and Z. Ren, "Enhancement of thermoelectric figure-ofmerit by a bulk nanostructuring approach," Advanced Functional Materials, vol. 20, pp. $357-376,2010$.

9. R. Venkatasubramanian, E. Siivola, T. Colpitts, and . O'Quinn, "Thin-film thermoelectric devices with high room-temperature figures of merit," Nature, vol. 413, pp. 597-602, 2001.

10. A. Majumdar, "THERMOELECTRIC DEVICES Helping chips to keep their cool," Nature Nanotechnology, vol. 4, pp. 214-215, 2009.

11. R. Venkatasubramanian, C. Watkins, C. Caylor, and G. Bulman, "Microscale Thermoelectric Devices for Energy Harvesting and Thermal Management," Technical Digest, Power $M E M S$, vol. 1, 2006.

12. K. Fukutani and A. Shakouri, "Design of bulk thermoelectric modules for integrated circuit thermal management," IEEE Transactions on Components and Packaging Technologies, vol. 29, pp. 750-757, 2006.

13. Y. Ezzahri, G. Zeng, K. Fukutani, Z. Z. Bian, and A. Shakouri, "A comparison of thin film microrefrigerators based on $\mathrm{Si} / \mathrm{SiGe}$ superlattice and bulk SiGe," Microelectronics Journal, vol. 39, pp. 981-991, 2008.

14. L. Anatychuk, "Current Status and Some Prospects of Thermoelectricity," 5th European conference on thermoelectricity, 2007.

15. L. E. Bell, "Cooling, heating, generating power, and recovering waste heat with thermoelectric systems," Science, vol. 321, pp. 1457-1461, 2008.

16. J. Bierschenk and D. Johnson, "Extending the limits of air cooling with thermoelectrically enhanced heat sinks," pp. 679-684, 2004.

17. C. Jeong, R. Kim, M. Luisier, S. Datta, and M. Lundstrom, "On Landauer versus Boltzmann and full band versus effective mass evaluation of thermoelectric transport coefficients," Journal of Applied Physics, vol. 107, no. 2, p. 023707, 2010.

18. R. Kim, S. Datta, and M. S. Lundstrom, "Influence of dimensionality on thermoelectric device performance," Journal of Applied Physics, vol. 105, no. 3, p. 034506, 2009.

19. G. Chen and A. Shakouri, "Heat transfer in nanostructures for solid-state energy conversion," Journal of Heat Transfer, vol. 124, pp. 242-253, 2002.

20. P. N. Martin, Z. Aksamija, E. Pop, and U. Ravaioli, "Reduced thermal conductivity in nanoengineered rough ge and gaas nanowires." Nanoletters, ACS, vol. 10, pp. 1120-4, 2010 .

21. R. Landauer, "Spatial variation of currents and fields due to localized scatterers in metallic conduction," IBM J. Res. Dev., vol. 1, no. 3, pp. 223-231, 1957.

22. S. Datta, "Electronic Trasport in Mesoscopic Systems," Cambridge University Press, p. 393, 1997.

23. C. Jeong, S. Datta, and M. Lundstrom, "Full dispersion versus debye model evaluation of lattice thermal conductivity with a landauer approach," Journal of Applied Physics, vol. 109, no. 7, 2011. 
24. F. Zahid and R. Lake, "Thermoelectric properties of $B i_{2} T e_{3}$ atomic quintuple thin films," Applied Physics Letters, vol. 97, p. 212102, 2010.

25. N. Neophytou, M. Wagner, H. Kosina, and S. Selberherr, "Analysis of Thermoelectric Properties of Scaled Silicon Nanowires Using an Atomistic Tight-Binding Model," Journal of Electronic Materials, vol. 39, pp. 1902-1908, 2010.

26. N. Mingo, L. Yang, D. Li, and A. Majumdar, "Predicting the thermal conductivity of si and ge nanowires," Nano Letters, vol. 3, no. 12, pp. 1713-1716, 2003.

27. N. Mingo and L. Yang, "Phonon transport in nanowires coated with an amorphous material: An atomistic Green's function approach," Phys. Rev. B, vol. 68, no. 24, p. 245406, Dec 2003.

28. J. O. Sofo and G. D. Mahan, "Thermoelectric figure of merit of superlattices," vol. 65, no. 21 , pp. 2690-2692, 1994.

29. P. E. Blöchl, O. Jepsen, and O. K. Andersen, "Improved tetrahedron method for brillouinzone integrations," Phys. Rev. B, vol. 49, pp. 16 223-16 233, Jun 1994.

30. M. Methfessel and A. T. Paxton, "High-precision sampling for brillouin-zone integration in metals," Phys. Rev. B, vol. 40, pp. 3616-3621, Aug 1989.

31. A. Paul, M. Luisier, and G. Klimeck, "Modified valence force field approach for phonon dispersion: from zinc-blende bulk to nanowires," Journal of Computational Electronics, vol. 9, pp. 160-172, 2010.

32. - "Atomistic modeling of the phonon dispersion and lattice properties of free-standing $i^{100} i$ Si nanowires," 14th IEEE Workshop of Comp. Elect. DOI:10.1109/IWCE.2010.5677959, 2010.

33. H. Peelaers, B. Partoens, and F. M. Peeters, "Phonon Band Structure of Si Nanowires: A Stability Analysis," Nano Letters, vol. 9, no. 1, pp. 107-111, 2009.

34. "Workspace," Apr 2006. [Online]. Available: http://nanohub.org/resources/1242

35. S. Lee and P. von Allmen, "Tight-binding modeling of thermoelectric properties of bismuth telluride," Applied Physics Letters, vol. 88, no. 2, p. 022107, 2006.

36. H. J. Goldsmid, "Thermoelectric Refrigeration," Plenum Press, 1964.

37. J. D. Gale and A. L. Rohl, "The General Utility Lattice Program (GULP)," Mol. Simul., vol. 29, p. 291, 2003.

38. P. A. Walker, "The Thermal Conductivity and Thermoelectric Power of Bismuth Telluride at Low Temperatures," Proc. Phys. Soc., vol. 76, pp. 113-126, 1960. 


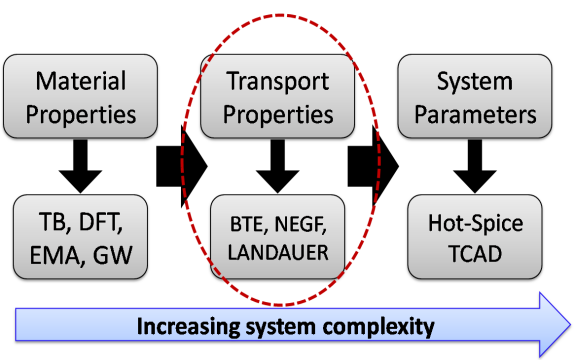

Fig. 1 Modeling hierarchy for the thermoelectric analysis. The present work focuses on the calculation of the TE parameters from the energy dispersion relations as shown by the encircled part.

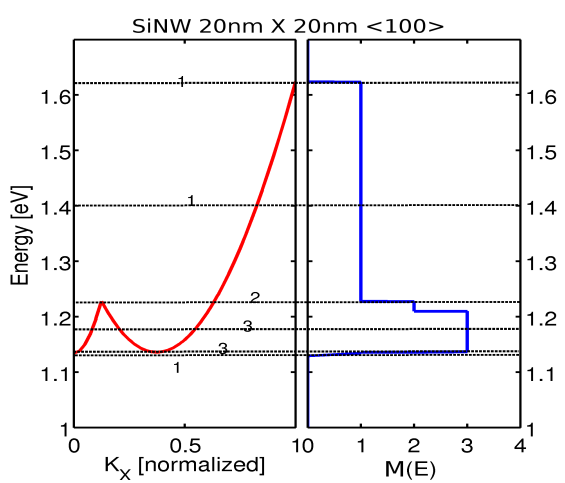

Fig. 2 The band-counting method for calculating the DOM. (a) The lowest sub-band of the electronic $\mathrm{E}(\mathrm{k})$ of a $20 \mathrm{~nm} \times 20 \mathrm{~nm}[100]$ SiNW. (b) The corresponding propagating modes $\mathrm{M}(\mathrm{E})$ associated with this single band. 

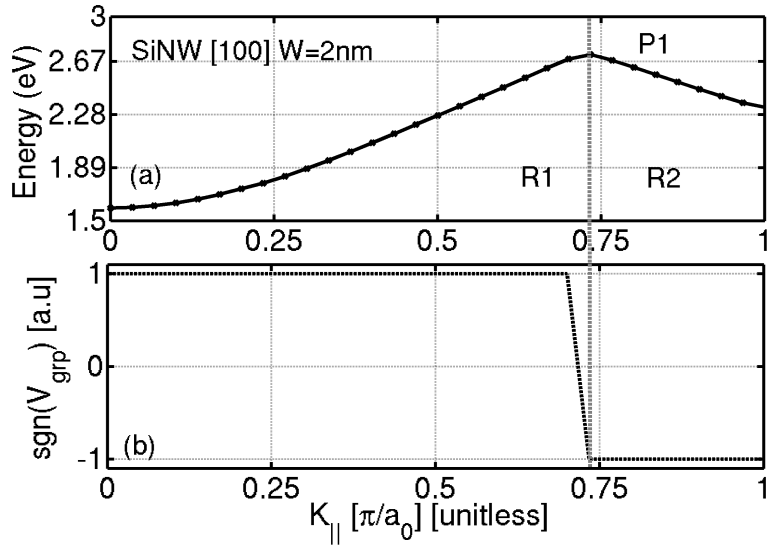

Fig. 3 Velocity at each point positive half of first conduction band sub-band for a $2 \mathrm{~nm} X$ $2 \mathrm{~nm}$ [100] Si nanowire (SiNW). As mentioned above, the important points to note in the given band are the points where sign of velocity changes. These points are indicated as P1, P2, and P3 and corresponding regions of interest are marked as R1, R2, and R3 in Figure 2.3. Each point provides monotonic velocity range (increasing or decreasing) and calculations for DOM are performed separately for these ranges within a sub-band.

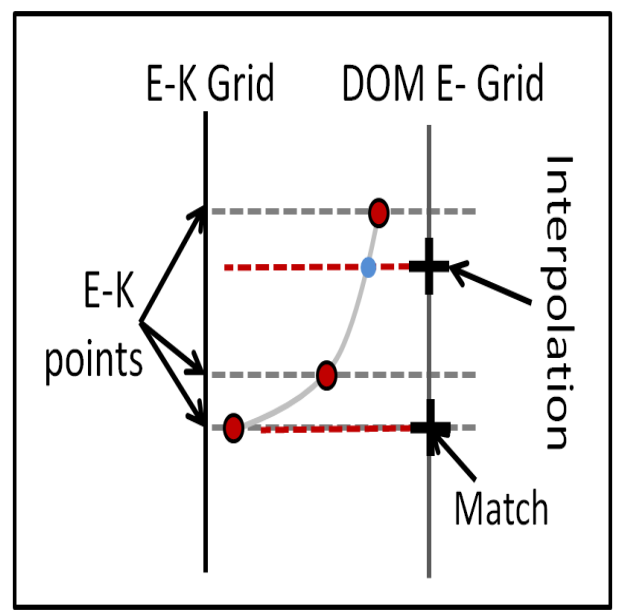

Fig. 4 The $E-K$ points on the provided energy dispersion shown by dots. The energy grid for DOM (EGD) points are shown using crosses. These points are of two types, (i) the matching point to the $E-K$ grid, and (ii) the one which requires interpolation of the provided $E-K$ relationship. This interpolation (either linear or quadratic) is done in the appropriate monotonic energy region like $R_{1}, R_{2}$, etc shown in Fig. 3 In this way the DOM is created for the EGD. 


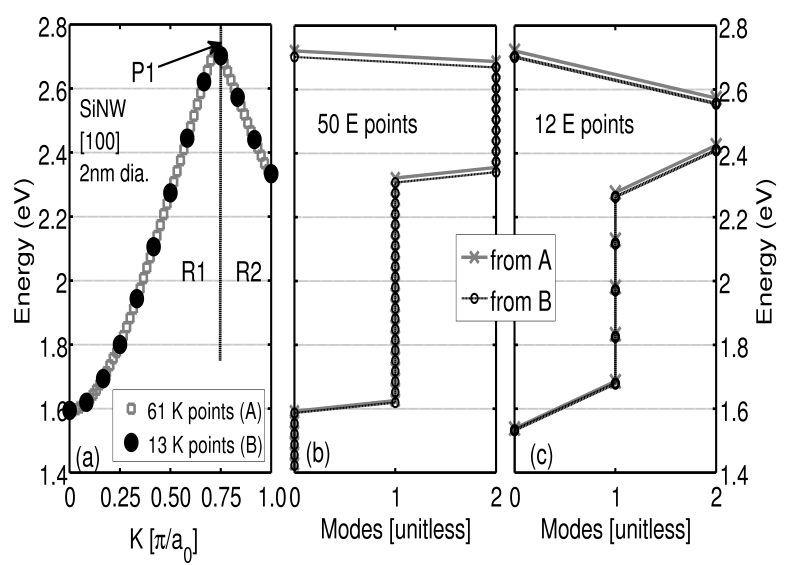

Fig. 5 (a) $E(K)$ relation with different number of k points. Case A with $61 \mathrm{~K}$ points and case $\mathrm{B}$ with $13 \mathrm{~K}$ points. Also the two monotonic $E-K$ regions are shown (R1 and R2) along with the turn around point P1. DOM calculated for the two $E-K$ grids using (b) 50 energy points and (c) 12 energy points. The DOM matches $100 \%$ for all the 4 cases showing the robustness of the DOM calculation method. As long as the sparse $E-K$ captures the important turn around points (like P1) correctly the DOM calculation algorithm obtains the correct number of modes.

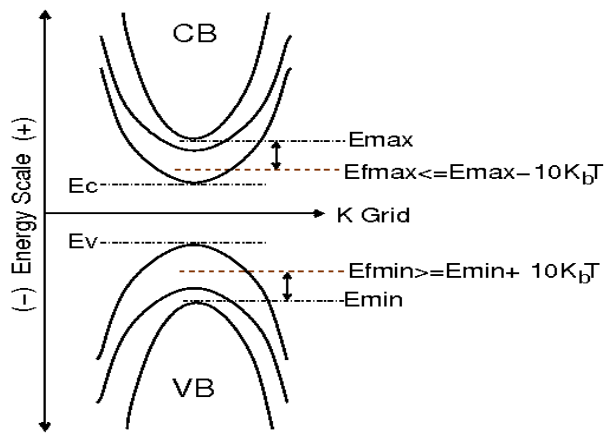

Fig. 6 Schematic showing the range of energy limit and the range of Fermi-level used for the calculation of the integral in Eq. 6 for electrons. 

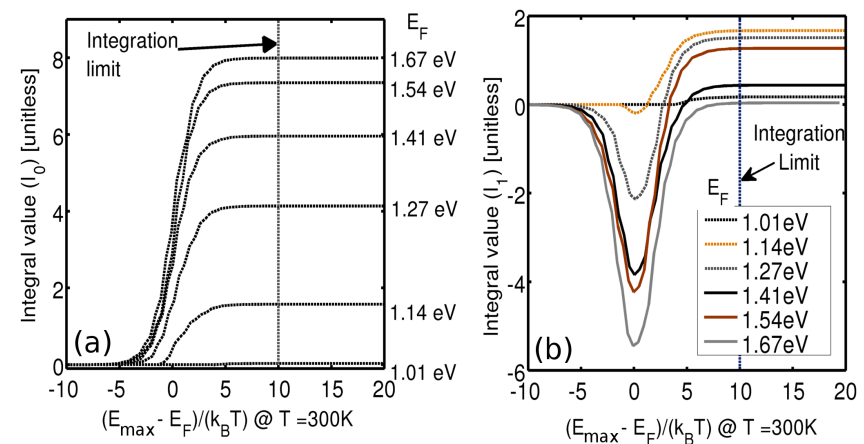

Fig. 7 Variation in the values of (a) $I_{0}$ and (b) $I_{1}$ (Eq. 6 for different values of the Fermi-level $\left(E_{F}\right)$. When the $E_{F}$ is at least $10 k_{B}$ T below the $E_{\max }$ then the integral values show less than $1 \%$ variation. Similar result is also obtained for the integral $I_{2}$.
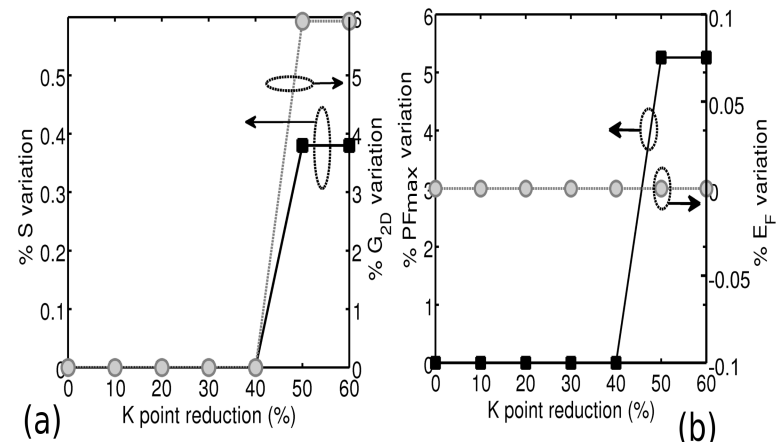

Fig. 8 Impact of $K_{\|}$point reduction on (a) $S$ (left) and $G$ (right) and (b) $P F$ (left) and $E_{F}$ (right) in a 2D structure. All the values are extracted at the maximum PF point. The $K_{\perp}$ has 100 grid points. Even for $60 \%$ reduction in $K_{\|}$points none of the TE values show more than $6 \%$ variation.

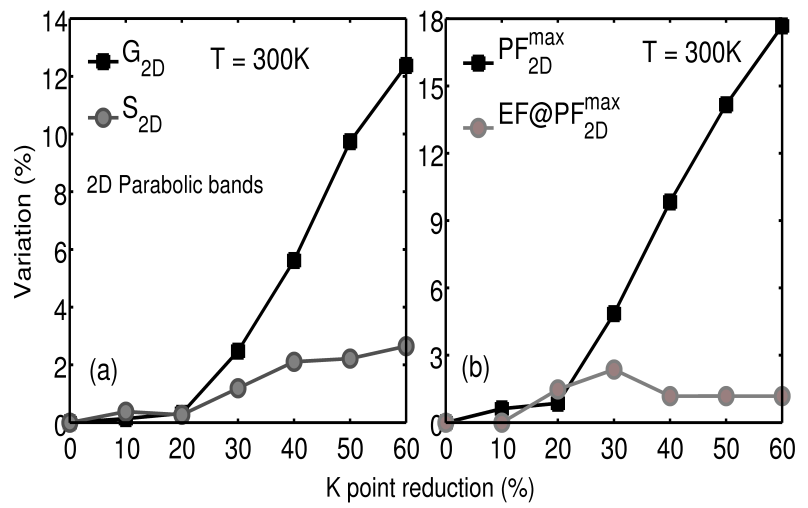

Fig. 9 Impact of $K_{\perp}$ point reduction on (a) $S$ (left) and $G$ (right) and (b) $P F$ (left) and $E_{F}$ (right) in a $2 \mathrm{D}$ structure. All the values are extracted at the maximum PF point. The $K_{\|}$ has 100 grid points. For $60 \%$ reduction in K-points $G$ shows a maximum variation of $12 \%$ and $P F_{\max }$ has variation around $10 \%$. 


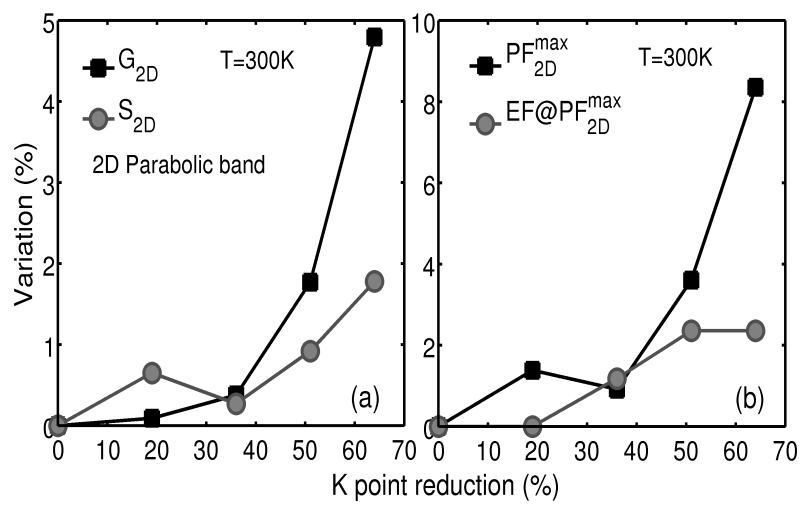

Fig. 10 Impact of reduction in all the K-points, on (a) $S$ (left) and $G$ (right) and (b) $P F$ (left) and $E F$ (right) in a 2D structure. All the values are extracted at the maximum PF point. $G$ shows a larger fluctuation $(\geq 10 \%)$ compared to $S$ fluctuation $(\leq 4 \%)$ which also reflects in the $P F_{\max }$ fluctuation.

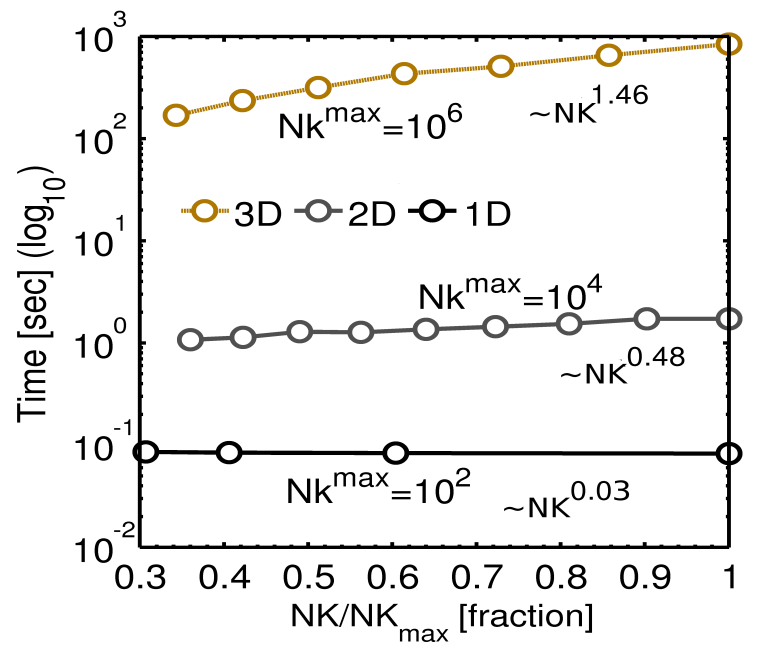

Fig. 11 DOM compute time for 1D, 2D and 3D parabolic bands. The number of $\mathrm{K}$ points are reduced along all the K-directions equally. The 3D case takes the maximum time due to higher number of K-points, followed by the 2D and the $1 \mathrm{D}$ case. 

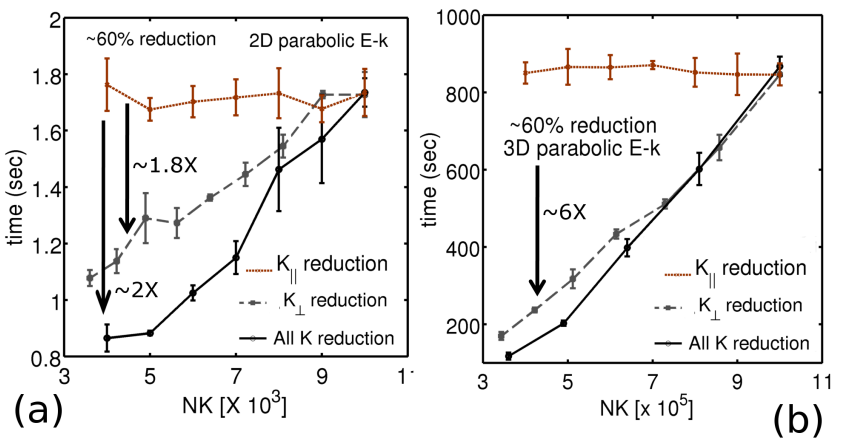

Fig. 12 DOM compute time $\left(t_{D O M}\right)$ for the three types of K-point reduction for the (a) $2 \mathrm{D}$ structure and (b) 3D structure. For both the cases the compute time is almost independent of $K_{\|}$reduction (brown line). reduces with $K_{\perp}$ point reduction. For each point 5 compute times are averaged. 

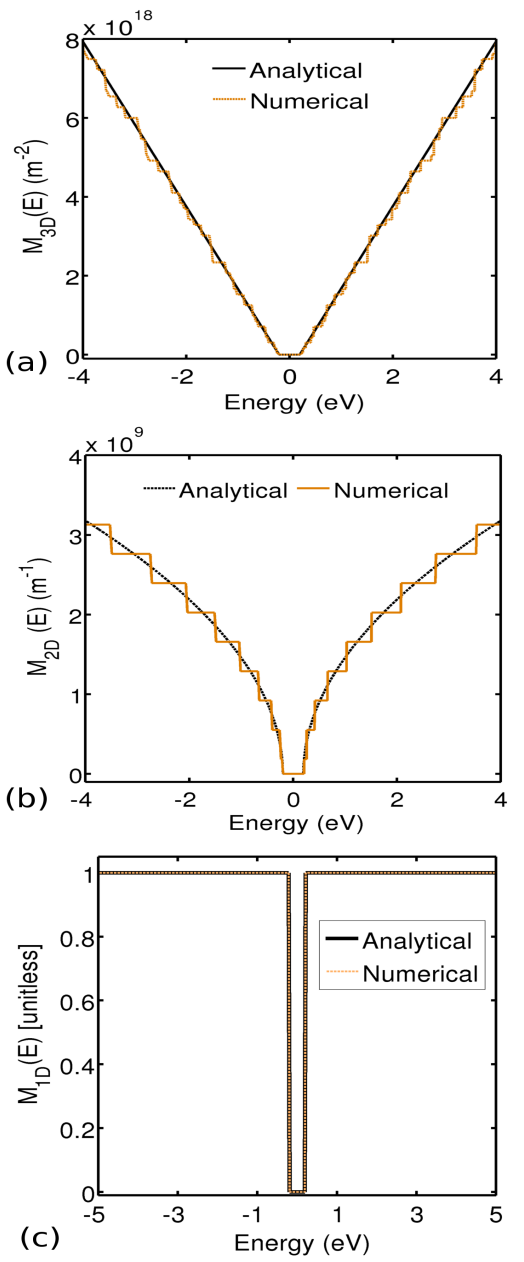

Fig. 13 Comparison of the numerical modes calculation using the algorithm with analytical modes calculation using parabolic bands with $m^{*}=m_{0}$ (from Ref [18]) for (a) 3D, (b) 2D and (c) $1 \mathrm{D}$ structure. The steps in the $2 \mathrm{D}$ case appear due to the sparse energy grid chosen. 

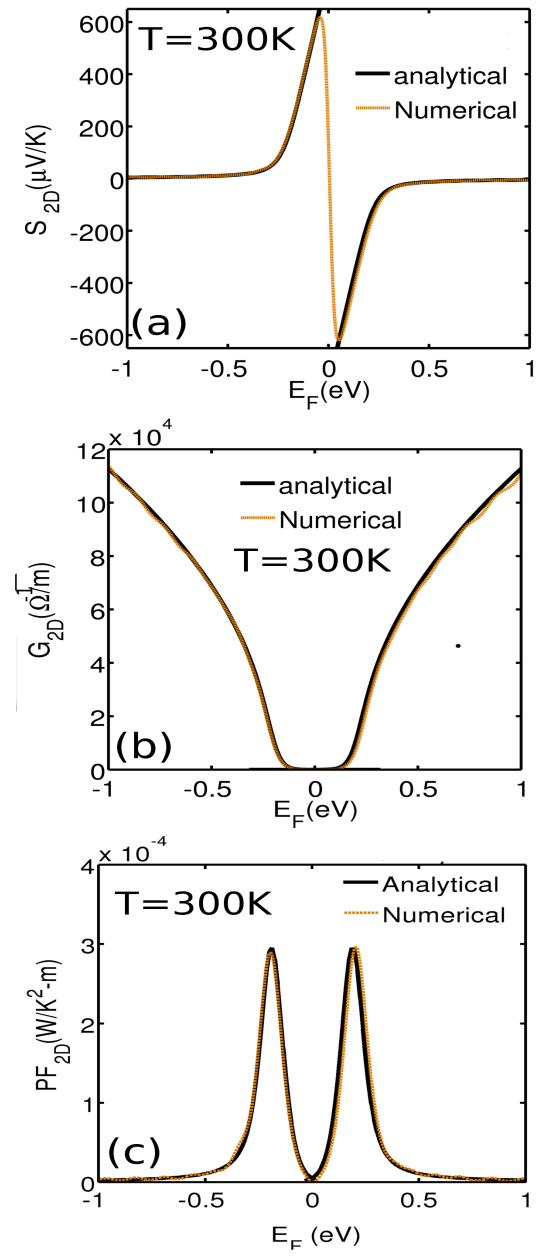

Fig. 14 Comparison of the numerical calculation with analytical expression for effective mass from Ref 18 for a 2D system for (a) Conductance (b) Seebeck Coefficient and (c) Thermoelectric Power Factor at 300K. The numerical results compare within $1 \%$ to the analytical values. The parameters used for the parabolic bands are provided in Table 2 


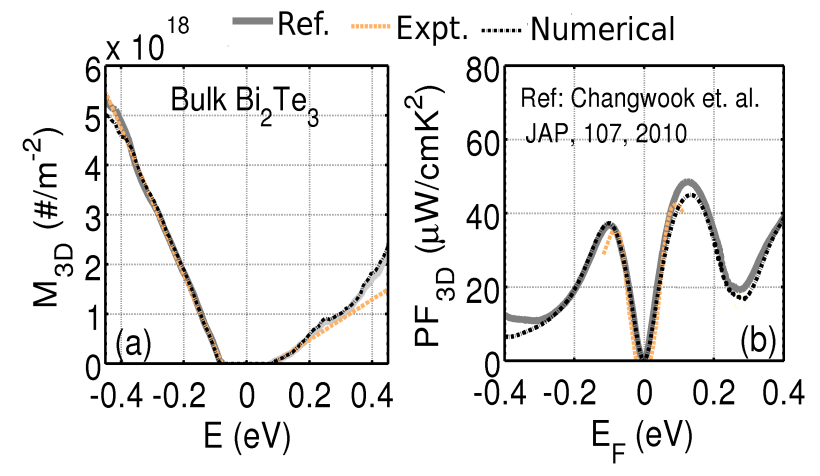

Fig. 15 Comparison of (a) DOM calculation and (b) Power factor at $300 \mathrm{~K}$ for $B i_{2} \mathrm{Te}_{3}$, using the algorithm, with the theoretical calculations reported in Ref. 17 and experimental results from [36]. The electronic energy dispersion for bulk $B i_{2} T e_{3}$ is obtained using the $s p^{3} d^{5} s^{*}$ tight-binding model 35. The PF matching for $B i_{2} \mathrm{Te}_{3}$ is obtained assuming a constant meanfree-path of 18, $4 \mathrm{~nm}$ for conduction and valence bands respectively as reported in Ref. [17.
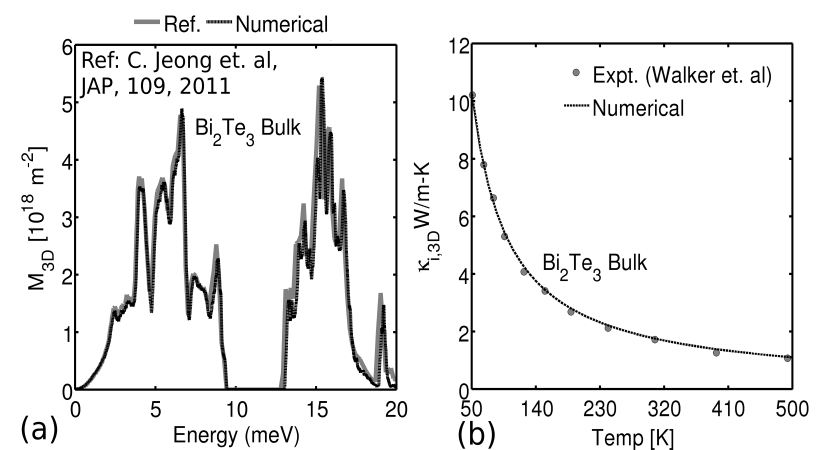

Fig. 16 (a) Comparison of the bulk $B i_{2} T e_{3}$ phonon modes calculated using the algorithm and theoretical value reported in Ref. 23. The bulk phonon dispersion is obtained using GULP 37. (b) Comparison of the simulated and experimental 38 thermal conductivity for Bulk $\mathrm{Bi}_{2} \mathrm{Te}_{3}$ from 50 to $500 \mathrm{~K}$. The phonon scattering mechanisms considered here are outlined in detail in Ref. 23. 